\title{
Clozapine Modulates Glucosylceramide, Clears Aggregated Proteins, and Enhances ATG8/LC3 in Caenorhabditis elegans
}

\author{
Limin Hao*,', Oshrit Ben-David², Suzann M Babb', Anthony H Futerman², Bruce M Cohen' and \\ Edgar A Buttner' \\ 'Program for Neuropsychiatric Research, Mailman Research Center 331, McLean Hospital, Belmont, MA, USA; ${ }^{2}$ Department of Biological \\ Chemistry, Weizmann Institute of Science, Rehovot, Israel
}

\begin{abstract}
Defining the mechanisms of action of the antipsychotic drug (APD), clozapine, is of great importance, as clozapine is more effective and has therapeutic benefits in a broader range of psychiatric disorders compared with other APDs. Its range of actions have not been fully characterized. Exposure to APDs early in development causes dose-dependent developmental delay and lethality in Caenorhabditis elegans. A previous genome-wide RNAi screen for suppressors of clozapine-induced developmental delay and lethality revealed 40 candidate genes, including sms-I, which encodes a sphingomyelin synthase. One sms-I isoform is expressed in the C. elegans pharynx, and its transgene rescues the sms-I mutant phenotype. We examined pharyngeal pumping and observed that clozapine-induced inhibition of pharyngeal pumping requires sms-I, a finding that may explain the role of the gene in mediating clozapine-induced developmental delay/ lethality. By analyzing multiple enzymes involved in sphingolipid metabolism, and by observing the effect of addition of various lipids directly to the worms, we suggest that glucosylceramide may be a key mediator of the effects of clozapine. We further observed that clozapine clears protein aggregates, such as $\alpha$-synuclein, PolyQ protein, and $\alpha$ - I-antitrypsin mutant protein. In addition, it enhances ATG8/LC3. We conclude that clozapine appears to affect the development and induce lethality of worms, in part, through modulating glucosylceramide. We discuss the possible connections among glucosylceramide, protein aggregate clearance, and autophagy. Interactions, including mechanistic pathways involving these elements, may underlie some of the clinical effects of clozapine.

Neuropsychopharmacology (2017) 42, 951 -962; doi:I0.1038/npp.2016.230; published online 26 October 2016
\end{abstract}

\section{INTRODUCTION}

The first antipsychotic drugs (APDs) were discovered in the 1950s (Deng et al, 2008; Lieberman et al, 2008). Clinically, the direct mechanism of action of these drugs has been thought to be full or partial antagonism of monoamine receptors, especially dopamine D2 and serotonin receptors, the blockade of which leads, directly and indirectly, to widespread alterations in neuroconnectivity and neurotransmission (Brunello et al, 1995). Clozapine was the first of the atypical APDs (agents not primarily targeting dopamine D2 receptors) to be developed, and it is unusually effective for a broad range of syndromes, including treatment-resistant schizophrenia (Crilly, 2007). However, it has numerous side effects, rarely causing agranulocytosis, seizures, or myocarditis, but commonly causing less serious side effects such as sedation, hypersalivation dyslipidemias, and weight gain (Gohlke et al, 2012).

The distinct properties of clozapine and its multiple side effects suggest that in addition to its capability to inhibit

\footnotetext{
*Correspondence: Dr L Hao, Program for Neuropsychiatric Research, Mailman Research Center 33I, McLean Hospital, II5 Mill Street, Belmont, MA 02478, USA, Tel: + I 617855 3503, Fax: +617 8553670 , E-mail: liminhao1969@yahoo.com

Received 22 March 2016; revised 27 August 2016; accepted 21 September 2016; accepted article preview online 6 October 2016
}

neuroreceptors, including those for dopamine, serotonin, norepinephrine, acetylcholine, and histamine, such as other APDs, it may target additional sites and regulate other biological processes. Clozapine has been shown to dimerize dopamine receptors and 5-HT2A receptors, which might modify their subcellular localization, trafficking, or interaction with proteins (Lukasiewicz et al, 2011). It has also been shown to balance the activity of $G$ proteins binding to 5-HT2A and mGluR2 receptors and thus modulate signaling output and behavioral changes (Fribourg et al, 2011).

To further understand the targets and action of clozapine, we used Caenorhabditis elegans as a physiological and genetic model. C. elegans is useful for these studies because molecular processes and drug effects in worms are often comparable to those observed in other organisms, including humans (Dwyer et al, 2014). Clozapine delays the development of $C$. elegans and, depending on the drug concentration used, can cause lethality. It also alters worm behavior, including decreasing the rate of pharyngeal pumping, inducing faster egg laying, and increasing the speed of locomotion (Donohoe et al, 2006; Karmacharya et al, 2011). The insulin/PKT pathway (which is altered in schizophrenia) has a role in clozapine-induced development delay/lethality, but it does not fully explain the effects of clozapine (Karmacharya et al, 2009). Clozapine is also involved in 
modulating serotonin-controlled behavior (Donohoe et al, 2009).

C. elegans is well suited for genomic studies to identify the mechanisms of disease and drug response. In our previous whole-genome wide RNA screen for suppressors of clozapine-induced development delay/lethality, we found 40 genes related to clozapine effects (Saur et al, 2013). Two of these genes, acr-7, which codes for an acetylcholine receptor, and transient receptor potential melastatin (TRPM) have already been suggested as possible targets of clozapine (Saur et al, 2013; Wang et al, 2014). In the current study, we present evidence that another suppressor, $s m s-1$, coding for sphingomyelin (SM) synthase 1, is a strong suppressor of clozapine-induced effects. It largely relieves the developmental delay/lethality phenotype when the gene is knocked down by RNAi.

$\mathrm{SM}$ is a sphingolipid (SL) found at high levels in the myelin sheaths that surround axons. It consists of a phosphorylcholine head group attached to a ceramide backbone. The synthesis of SM from ceramide is catalyzed by SM synthase in the Golgi apparatus (Futerman et al, 1990). Ceramide is both a precursor and a breakdown product of SM (and other SLs), and has a number of important signaling and regulatory functions (Gault et al, 2010), including modulation of autophagy and apoptosis (Gault et al, 2010; Harvald et al, 2015).

The brain contains high levels of lipids, including SLs, and irregularities of their metabolism often results in pathology (Futerman, 2015). Abnormalities in SL metabolism have been associated with psychiatric and neurodegenerative disorders (Narayan and Thomas, 2011), including reductions in phosphatidylcholine, SM, and galactocerebrosides in postmortem brains from subjects with schizophrenia (Schmitt et al, 2004). A high-throughput lipidomic profiling of schizophrenia and bipolar disorder brain tissue revealed that ceramides are increased in white matter of both schizophrenia and bipolar patients (Schwarz et al, 2008). As to neurodegenerative disorders, ceramides in sera have been associated with an increased risk of Alzheimer's disease (AD) (Mielke et al, 2012). The acid sphingomyelinase/ceramide system has been shown to be important in pathogenesis, as accumulation of ceramide in the hippocampus results in depression-like symptoms (Gulbins et al, 2013; Jernigan et al, 2015). Furthermore, a pilot study demonstrated that plasma ceramide and glucosylceramide (GlcCer) metabolism is altered in the sporadic Parkinson's disease (Mielke et al, 2013).

Clozapine can alter brain membrane phospholipid levels. In rat brain, clozapine activates carnitine palmitoyl transferase 1 (CPT1) through the AMP-activated protein kinaseacetyl CoA carboxylase signaling pathway (Kim et al, 2012). Relevant to this finding, Virmani et al (2015) have reviewed evidence that CPT1 affects ceramide levels in the brain and may be linked to psychiatric disorders, including schizophrenia.

We now directly investigate the effect of clozapine on SL metabolism and observe that GlcCer most likely mediates the developmental effects of clozapine in C. elegans. In addition, we also observe that clozapine clears protein aggregates and enhances ATG8/LC3, which may imply a relationship among glucosylceramide, autophagy, and protein aggregates.

\section{MATERIALS AND METHODS}

\section{Nematode Growth and Worm Strains}

The nematodes were cultured on NGM plates in a $20^{\circ} \mathrm{C}$ incubator (Lewis and Fleming, 1995) and operated at room temperature $\left(21-22^{\circ} \mathrm{C}\right)$. The wild-type $\mathrm{N} 2$ worm was used in these experiments as a control species. Other worm strains used are listed as follows: EAB1: sms-1(ok2399); EAB2: sms-1 (tm2666); EAB3: sms-1(tm2660); sms-2(tm2613); RB2549: sms-3(ok3540); CB30: sma-1(e30); CB6627: srf-3(e2689); RB1036: hyl-1(ok96); RB1498: hyl-2(ok1766); VC765: lagr-1 (gk331); VC916: sphk-1(ok1097); tm5023: asm-1; tm3746: asm-2; RB1487: asm-3(ok744); VC242: tag-38(ok490); RB2135: F33D4.4(ok2843); RB1465: sptl-1(ok1693); RB1579: sptl-3(ok1927); RB782: F27E5.1(ok564); RB1203: T10B11.2 (ok1252); tm504: cgt-3; HY483: bre-3(ye26); HY498: bre-5 (ye17); HY485: bre-4(ye27); HY494: bre-2(ye31); XR5: lagr-1 (gk327); hyl-1(ok976);ced-3(n717); tm3816: gba-1; tm3302: gba-3; VC3135: gba-3(gk3287); tm3314: gba-4; tm3349: gba4; DA2123(adIs2122): (lgg-1p::GFP::lgg-1+rol-6(su1006); NL5901(pkIs2386): unc-54p::alpha-synnuclein::YFP+unc119(+); VK689(vkIs689): nhx-2p::sGFP::ATM+myo-2p:: mCherry; VK694(vkIs694): nhx-2p::sGFP::ATZ; myo-2p:: mCherry; VK1241(vkEx1241): nhx-2p::mCherry::lgg-1; myo-2p::GFP; DA2123(adIs2122): lgg-1p::GFP::lgg-1+rol-6 (su1006); AM141(rmIs133): unc-54p::Q40::YFP.

\section{Sequence Analysis, Molecular Cloning, and Generation of Transgenic Worms}

The nematode SMS-1, SMS-2, and SMS-3 protein sequences were obtained from WormBase (http://www.wormbase.org), and the human SMS-1 protein sequence was obtained from NCBI BLAST (http://blast.ncbi.nlm.nih.gov/Blast.cgi) by using $C$. elegans SMS-1 as the query sequence. The alignments were performed by CLUSTAL X2.

The $s m s-1$ gene was predicted to have six isoforms. They could be divided into three groups, which shared the same putative promoters. For the transcriptional constructs, the approximate $2-3 \mathrm{~kb}$ upstream sequences of each putative promoter were cloned into the pPD95.67 vector containing the GFP sequence. As to the translational constructs, either the sms-1 cDNA or genomic sequences were added into the transcriptional constructs and fused with GFP.

To generate the transgenic worms, the standard worm injection technique was used. The transcriptional constructs were injected into the wild-type worms to observe the gene expression patterns, and the translational constructs were injected into $s m s-1(0 k 2399)$ mutant worms for the rescue experiments. The dominant roller gene rol-6 (in the plasmid pRF4) was used as the injection marker.

\section{Developmental Assay and Pharyngeal Pumping Assay}

The methods of the developmental assay have been detailed in (Hao and Buttner, 2014). Briefly, the well-grown adult animals were bleached using hypochlorite: $1 \mathrm{~N}$ $\mathrm{NaOH}: \mathrm{ddH}_{2} \mathrm{O}(1: 4: 5)$ to obtain synchronized eggs, 25 of which were then placed on the 12-well NGM assay plates containing variable concentrations of drugs. The drugs were initially dissolved in DMSO to obtain $80 \mathrm{mM}$ stock solutions, which were diluted into $1.7 \mathrm{mM}$ acetic acid solution to get 
the suspension solution, which was transferred onto the NGM plates. Afterwards, every $24 \mathrm{~h}$ the plates were observed and the developmental status was recorded. The assay usually lasted 5-7 days. The results on the third or fourth day produce the most obvious results and therefore those results were shown.

The assays were set up and developmental status was observed and recorded every day for 7 days. We only showed the results on the third day, because that observation usually distinguished when worms developed differently. For each strain, we performed the experiments on three batches of worms set up and studied on different dates for each worm strain. We mostly used the higher concentration of drug so that results could be obtained within 3 or 4 days. To address differences among worm strains, we adjusted concentrations of the drug, for example, for some clozapine-sensitive mutants, the drug concentrations were lowered. These procedures follow observations published in previous manuscripts (Karmacharya et al, 2009, 2011).

For pharyngeal pumping assay, the well-grown L4 worms were picked $24 \mathrm{~h}$ before the assay. The next day, 10 staged adult worms were placed on the NGM assay plates containing variable concentrations of drugs, and allowed to adapt for $30 \mathrm{~min}$. Then, pharyngeal pumping was counted for $20 \mathrm{~s}$ for each worm.

\section{Extraction of Total Lipids and SLs}

Total lipid and SLs were extracted and were adapted from Menuz et al (2009). The bacterial or worm samples $(\sim 0.3 \mathrm{ml})$ were ground in a mortar immersed in a dry ice and ethanol. The ground powder was transferred into a glass tube and stored on ice. When melted, three $10 \mu \mathrm{l}$ aliquots were taken and stored at $-20^{\circ} \mathrm{C}$ for protein determination (using the BCA Protein Assay Kit from Thermo Scientific). The remaining was mixed with four times of $\mathrm{CH}_{3} \mathrm{Cl}: \mathrm{CH}_{3} \mathrm{OH}$ $(2: 1(\mathrm{~V} / \mathrm{V}))$ vigorously, and then centrifuged at $200 \mathrm{~g}$ at $4{ }^{\circ} \mathrm{C}$ for $10 \mathrm{~min}$. The lower phase was recovered and 0.2 volume of double distilled water $\left(\mathrm{ddH}_{2} \mathrm{O}\right)$ was added and centrifuged again. The resulting organic phase was dried under the nitrogen gas, sealed, and stored at $-20^{\circ} \mathrm{C}$. For extraction of total lipids from bacterial strain OP50, the same procedure was applied.

For SL extraction, the total lipid preparation extracted from worms was dissolved in $600 \mu \mathrm{l} \mathrm{CHCl}: \mathrm{CH}_{3} \mathrm{OH}: \mathrm{ddH}_{2} \mathrm{O}$ $(16: 16: 5)$ to which $600 \mu \mathrm{l}$ of $0.2 \mathrm{~N} \mathrm{NaOH}$ (diluted in $\mathrm{CH}_{3} \mathrm{OH}$ ) was added. After incubation at $30{ }^{\circ} \mathrm{C}$ for $45 \mathrm{~min}$, $600 \mu \mathrm{l}$ of $0.5 \mathrm{M}$ EDTA was added, followed by $120 \mu \mathrm{l}$ of $1 \mathrm{~N}$ acetic acid. The mixture was centrifuged at $200 \mathrm{~g}$ for $10 \mathrm{~min}$. The lower phase was recovered and washed once with $\mathrm{dd}_{2} \mathrm{O}$, and then dried under the nitrogen gas and stored at $-20^{\circ} \mathrm{C}$.

\section{Lipid Rescue Assay}

For the lipid rescue assays, the total lipids or SLs from bacteria or worms were dissolved in DMSO and their concentrations were estimated based on the amount of the protein content in the starting material. The dissolved lipids were spotted onto the assay plates and allowed to dry before placing the eggs or worms.
Ceramides were obtained from Avanti Polar Lipids and Cayman Chemicals. The C-16:0, C-20:0, and C-22:0 ceramides were dissolved in $1 \% \mathrm{NP}-40$, and the C-24:0 ceramide was dissolved in methanol, all at $1 \mu \mathrm{g} / \mathrm{ml}$. For the rescue experiments, 15 and $30 \mu$ were supplemented onto the worm test plates. The combination of the ceramides was a mixture of the individual ceramide solutions.

\section{RESULTS}

\section{sms-1 with Reduced Activity Suppress Clozapine- Induced Developmental Delay/Lethality}

A previous genome-wide RNAi screen revealed that knockdown of sms-1 suppressed clozapine-induced developmental delay/lethality (Saur et al, 2013). The sms-1 gene has six putative transmembrane domains, conserved between nematodes and humans (Supplementary Figure S1). Three deletion mutant alleles of $s m s-1(o k 2399, t m 2666, t m 2660)$ are available. Drug assays demonstrated that only ok2399 shared the RNAi phenotype (Figure 1a), suggesting that the other two mutants might not be null. Notably, the mutations of tm2666 and $t m 2660$ deleted non-conserved protein regions, which might not reduce protein activity. However, the deletion region in ok2399 started from an intron and ended in an exon, so that it removed not only a small conserved protein region but also changed the splicing site of the intron, which likely leads to a reading frame shift following the deletion and thus complete loss of the protein sequence from TM3 to the end of the peptide (Figure $1 \mathrm{~b}$ and Supplementary Figure S1). The sms-1 gene has two orthologs, sms-2 and sms-3, in the C. elegans genome, knockouts of which did not suppress clozapine-induced developmental delay/lethality (Figure 1a). We determined other phenotypes of the sms-1(ok2399) mutant and found that it exhibited small brood size and Unc and Egl phenotypes (Supplementary Figure S2).

The six sms-1 gene isoforms can be grouped into three categories with three types of putative promoters. Correspondingly, we made three transcriptional and translational GFP fusion constructs (Figure 1b). The long isoform was expressed in the pharyngeal gland cells, spermacecae, and coelomocytes. The medium-sized isoform is expressed in all muscles including body wall muscle, head and tail muscles, vulval muscles, pharyngeal muscles, and anal muscles. The short isoform is only expressed in the ventral nerve cord (data not shown).

\section{An sms-1 Mutation that Relieves Clozapine-Induced Inhibition of Pharyngeal Pumping}

Three translational GFP fused transgenes were injected into sms-1(ok2399) worms for rescue experiments. The longest isoform, expressed in the pharyngeal gland and spermacecae (Figure 1c), rescued the clozapine-induced developmental delay/lethality phenotype (Figure 1d). The other two isoforms did not rescue the phenotype (data not shown).

As the rescuing isoform was expressed in the pharyngeal gland cells, we performed pharyngeal pumping assays to characterize gene-drug interactions. As shown previously, clozapine reduced the pharyngeal pumping rate in wild-type worms (Figure 1d). The mutation in sms-1(ok2399) relieved 
a

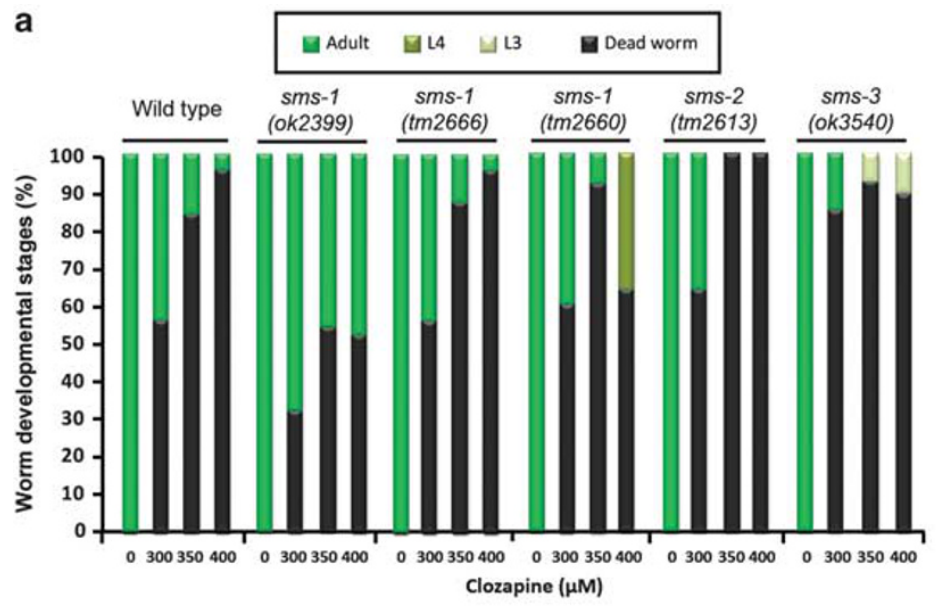

b

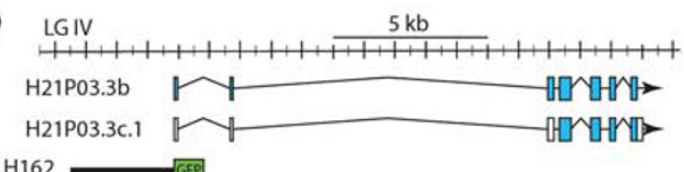

pLH162

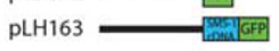

\section{H21P03.3a

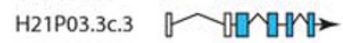

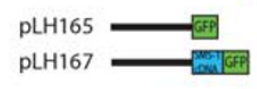

H21P03.3c.2 ㅁNHYIP H21P03.3C.4 마낸ㄱ pLH164 pLH160

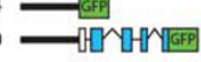
$\operatorname{tm} 2666$ I $\operatorname{tm} 2660=0 K 2399$ c
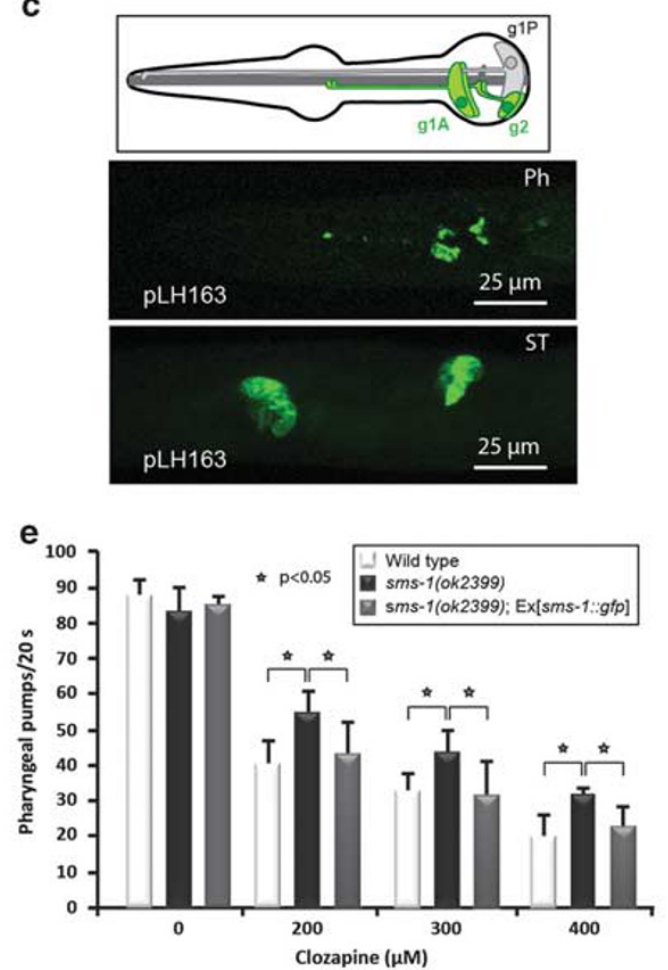

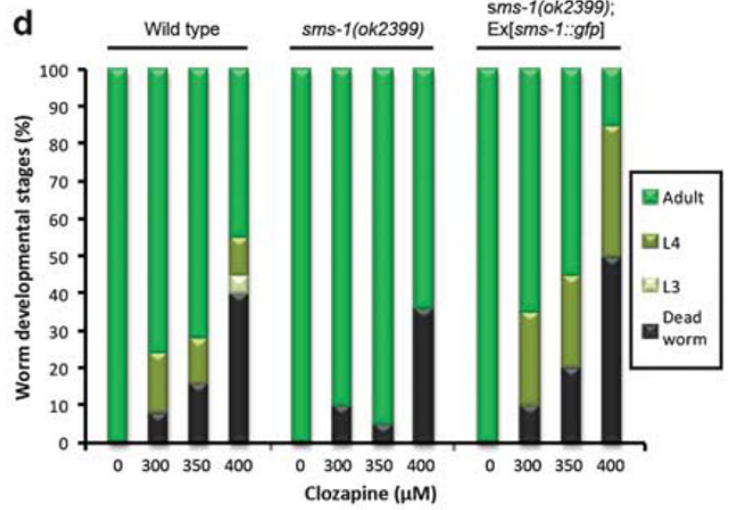

f

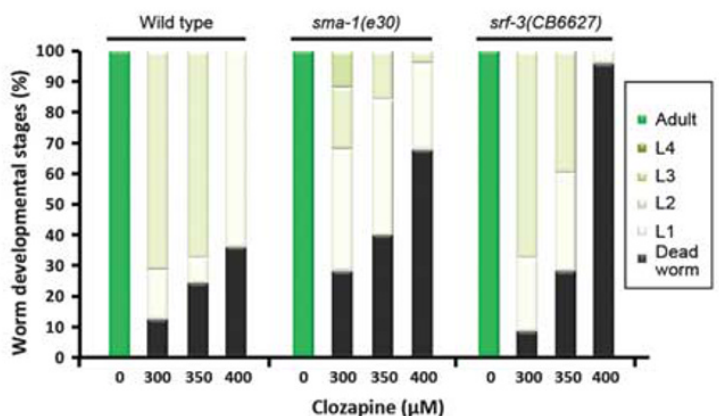

Figure I sms-I, a suppressor of clozapine-induced developmental delay/lethality and pharyngeal pumping rate decrease. (a) Three alleles of sms-I mutants (ok2399, tm2666, and tm2660) and two sms-I orthologs (sms-2 and sms-3) were tested using 300, 350, and $400 \mu M$ clozapine. The fraction of different developmental stages is shown. (b) The gene structure of sms-I and the GFP-fused transcriptional and translational constructs, and the three deletion alleles are shown. (c) Diagram of pharyngeal gland cells and location of SMSI::GFP onto the cell surface in the punctate shape. (d) The worms carrying sms-1:::ffp transgenes expressed in the pharyngeal gland cells, sms-I (ok2399) and wild type are treated with clozapine at 0, 300, 350, and 400 $\mu M$ and their developmental status are shown. (e) The worms carrying sms-1::gfp transgenes expressed in the pharyngeal gland cells, sms-I (ok2399) and wild type are treated with clozapine at 0, 200,300, and $400 \mu \mathrm{M}$ and their developmental status are shown. $N=10$, $t$-test is performed, and $* p<0.05$. ( $\mathrm{f}$ ) The mutant of sma- I, which disrupts the morphology of pharyngeal gland cells, and the mutant of srf-3, which affects the translocation of sugar into Golgi apparatus, are more sensitive to clozapine.

the drug-induced pumping rate decrease, and the sms-1 transgene reversed the relief effect of the sms-1(ok2399) mutant (Figure 1e).

To confirm the importance of gland cells in clozapineinduced developmental delay/lethality, we tested a mutant sma-1(e30), which had disrupted morphology of the gland cells and was hypersensitive to clozapine (Figure 1f). We also tested the other gene, $s r f-3$, that was expressed in pharyngeal gland cells and endowed the worms with resistance to infectious bacteria when mutated. This mutant was hypersensitive to clozapine (Figure 1f), suggesting that the pharyngeal gland cells do not provide a general protection for the animal.

The response of any worm strain to clozapine in independent experiments will differ somewhat among trials, for example, the lethality rate of wild-type strains shows 
some variance between experiments (Figures 1a and f). Such differences could arise from variability among batches of worms, even genetically identical worms, constitution of the assay plates, and could be a consequence of the dissolubility of clozapine in the media. Therefore, we only compared the results for the same batch of worms on the same batches of assay plates run at the same time.

\section{Glucosylceramide has a Role in Clozapine Effects}

The sms-1 gene encodes SM synthase, which converts ceramide and phosphatidylcholine into SM and diacylglycerol. Ceramide hss a central role in SL metabolism (Figure 2) and is synthesized by ceramide synthases (CerS), which are encoded by three genes in worms, hyl-1, hyl-2, and lagr-1. We compared ceramide and SM levels in sms-1 and hyl-1 worms by liquid chromatography-mass spectrometry, and preliminary results demonstrated that both ceramide and SM are decreased in hyl-1 and SM is decreased, but ceramide increased in $s m s-1$ (Supplementary Figure S3).

To investigate whether ceramide or SM may mediate the effects of clozapine, we conducted developmental and pharyngeal pumping assays on mutants of enzymes in the SL metabolic pathway. Mutants that potentially reduced ceramide levels (affecting the enzymes labeled in red in Figure 2) were more sensitive to clozapine, whereas those (labeled in blue in Figure 2) that potentially increased the levels of ceramide were more resistant to clozapine. These observations suggest that ceramide may have an important role in mediating the effects of clozapine.

Ceramides can be glycosylated to form glycosphingolipids (Barrows et al, 2007; Griffitts et al, 2005). We performed developmental and pharyngeal pumping assays on mutants of the glycosylation enzymes upon clozapine treatment. The mutant for the glucosylation enzyme (cgt-3), the first glycosylation step, was hypersensitive to clozapine compared with the wild type, whereas the mutant for the enzyme in the second glycosylation step was similar to wild type or somewhat more resistant to clozapine. Mutants in subsequent glycosylation steps were resistant to clozapine (Figure 2). These observations suggest that glucosylceramide is involved in mediating the effects of clozapine.

In the SL metabolism network, all the genes that potentially increase ceramide and GlcCer levels are hypersensitive to clozapine, thus we tested whether clozapine could lead to change in ceramide levels, and in the preliminary data found that most ceramide species were reduced in the clozapine-treated worms (Supplementary Figure S4). We also determined ceramide levels in PC12 cells, and found that the clozapine-treated PC12 cells at 10,20, and $40 \mu \mathrm{M}$ had reduced levels of ceramide species (Supplementary Figure S5).

We also explored whether clozapine can directly affect ceramide synthesis through effects on CerSs. The mammalian genes, CerS2 and CerS5, were overexpressed in PC12 cells and CerS activity assayed in vitro in the presence of clozapine, clozapine-N-oxide, or fumonisins B1 (a CerS inhibitor). Neither clozapine nor clozapine-N-oxide had any effect on CerS2 or CerS5 activity (Supplementary Figure S6).

\section{Ceramides Modulate Clozapine Effects}

We next performed rescue experiments using the strong enhancer phenotype of the hyl-1 mutant. We took advantage of the fact that most bacteria do not contain SLs (Sohlenkamp and Geiger, 2016) and thus used the common worm food bacteria (OP50 strain). First, we extracted total lipids from wild-type worms or bacteria and supplemented them into the drug assay plates for the hyl-1 mutant worms. Total lipids extracted from bacteria lacking SLs had no effects on clozapine-induced developmental delay/lethality (Figure 3a). However, total lipids extracted from wild-type worms, which do make SLs, relieved clozapine-induced developmental delay/lethality (Figure 3b), as did SLs extracted from wild-type worms (Figure 3c).

We next examined whether ceramides of defined acyl chain length could mediate the effects of clozapine. C-16:0, C-20:0, C-22:0, and C-24:0 ceramides individually were not capable of or had little effect on relieving clozapine effects, whereas the composite of a few ceramide species, C-16:0, C-20:0, C-22:0, and C-16:0, C-20:0, C-22:0, C-24:0, were able to relieve the effect of clozapine (Figure 3 and Supplementary Figure S7).

\section{Clozapine Reduces Protein Aggregates}

Gaucher disease patients carry mutations in glucocerebrosidase (GBA1), the enzyme that cleaves GlcCer in the lysosome; interestingly, patients with mutations in GBA1 have a higher risk of Parkinson's disease (Sidransky, 2005), which could conceivably be associated with reduced clearance of aggregated $\alpha$-synuclein. Some APDs clear aggregated proteins in C. elegans (Gosai et al, 2010; Li et al, 2014; McCormick et al, 2013). Thus, we examined whether clozapine was able to clear aggregated proteins, using a worm strain that carries YFP-labeled $\alpha$-synuclein driven by the unc-54 promoter expressed in muscles. In the adult, $\alpha$-synuclein aggregates form puncta (Figure $4 \mathrm{a}$ ), but in clozapine-treated worms, $\alpha$-synuclein is dispersed and the number of puncta dropped markedly (Figures $4 \mathrm{~b}-\mathrm{e}$ ). Clozapine also cleared other aggregated proteins, such as $\alpha$-1-antitrypsin mutant protein (ATZ) (Figures $4 \mathrm{f}-\mathrm{j}$ ) and PolyQ (Figures 4k-o).

\section{Clozapine Enhances lgg-1p::gfp::lgg-1}

As large protein aggregation can be cleared by autophagy, we tested whether clozapine affected autophagy. Transgenic worms carrying $\operatorname{lgg}-1 p:: g f p:: \operatorname{lgg}-1$ (Kang and Avery, 2009), where $\operatorname{lgg}-1$ is homologous to ATG-8 in other organisms, expressed the transgenic genes, and a labeled marker for autophagosomes was dispersed in the cell. There were fewer puncta, implying fewer autophagosomes (Figure 5a). After treatment with clozapine, the number of puncta was increased (Figures 5b-e), indicating that more autophagosomes were formed. To test whether the effect of clozapine might be mediated via GlcCer, we crossed the $\operatorname{lgg}-1 p:: g f p:: \operatorname{lgg}-1$ transgene into the cgt-3(tm504) mutant (Marza et al, 2009; Nomura et al, 2011), which has low GlcCer levels. Compared with that of wild-type strains, the number of puncta formed in the cgt-3(tm504) mutants was 


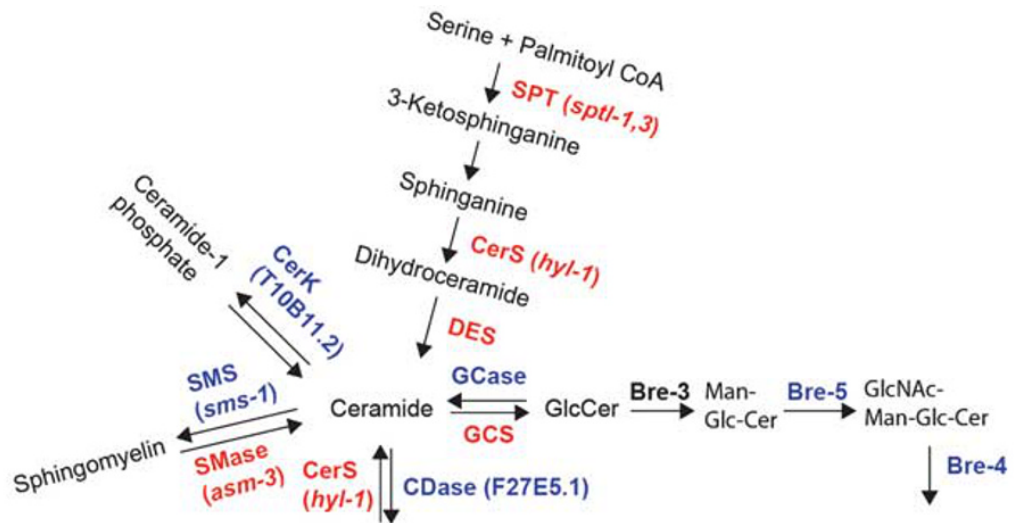

Sphingosine

$\downarrow$ SphK $($ sphk-1)

Sphingosine-1-phosphate (S1P)

$$
\downarrow \text { S1PPL (tag-38) }
$$

GaINAC-GICNAC-

Man-Glc-Cer

Long-chain aldehydrate

ethanolamine phosphate

$\downarrow$ Bre-2

GlanNAc-

GalNAcGICNAC--

Man-Glc-Cer

\begin{tabular}{|c|c|c|c|c|c|}
\hline Enzyme & Worm gene & Worm strain & Allele & $\begin{array}{l}\text { Developmental } \\
\text { delay/Lethality }\end{array}$ & $\begin{array}{l}\text { Pharyngeal } \\
\text { pumping }\end{array}$ \\
\hline \multirow{3}{*}{ SPT } & sptl-1 & RB1465 & ok1693 & Enhancer & Enhancer \\
\hline & sptl-2 & VC2358 & ok2753 & Wild type & Wild type \\
\hline & sptl-3 & RB1579 & ok1927 & Enhancer & Enhancer \\
\hline \multirow{3}{*}{ Cers } & hyl-1 & RB1036 & ok976 & Enhancer & Enhancer \\
\hline & hyl-2 & RB1498 & ok1766 & Wild type & Wild type \\
\hline & lagr-1 & VC765 & gk331 & Wild type & Wild type \\
\hline DES & F33D4.4 & RB2135 & ok2843 & Enhancer & -- \\
\hline Cerk & T10B11.2 & RB1203 & ok1252 & Suppressor & Suppressor \\
\hline \multirow{5}{*}{ SMS } & \multirow{3}{*}{$s m s-1$} & EAB1 & ok2399 & Suppressor & Suppressor \\
\hline & & tm2666 & $\operatorname{tm} 2666$ & Wild type & Wild type \\
\hline & & $\operatorname{tm} 2660$ & $\operatorname{tm} 2660$ & Wild type & Wild type \\
\hline & $s m s-2$ & $\operatorname{tm} 2613$ & $\operatorname{tm} 2613$ & Wild type & Wild type \\
\hline & $s m s-3$ & RB2549 & ok3540 & Wild type & Wild type \\
\hline \multirow{3}{*}{ SMase } & asm-1 & $\operatorname{tm} 5023$ & $\operatorname{tm} 5023$ & Wild type & Wild type \\
\hline & asm-2 & $\operatorname{tm} 3746$ & $\operatorname{tm} 3746$ & Wild type & Wild type \\
\hline & asm-3 & RB1487 & ok1744 & Enhancer & Enhancer \\
\hline CDase & F27E5.1 & RB782 & ok564 & Suppressor & Suppressor \\
\hline SphK & sphk-1 & VC916 & ok1097 & Suppressor & Suppressor \\
\hline S1PPL & B0222.4 & VC242 & ok490 & Suppressor & Suppressor \\
\hline \multirow{2}{*}{ GCS } & cgt-1 & VC693 & ok1045 & Wild type & Wild type \\
\hline & cgt-3 & $\operatorname{tm} 504$ & $\operatorname{tm} 504$ & Enhancer & Enhancer \\
\hline \multirow{5}{*}{ GCase } & gba-1 & $\operatorname{tm} 3816$ & $\operatorname{tm} 3816$ & Suppressor & - \\
\hline & \multirow{2}{*}{ gba-3 } & VC3135 & gk3287 & Wild type & -- \\
\hline & & $\operatorname{tm} 3302$ & $\operatorname{tm} 3302$ & Wild type & -- \\
\hline & \multirow{2}{*}{$g b a-4$} & $\operatorname{tm} 3314$ & $\operatorname{tm} 3314$ & Wild type & -- \\
\hline & & $\operatorname{tm} 3349$ & $\operatorname{tm} 3349$ & Wild type & - \\
\hline $\begin{array}{l}\beta \text {-glycosyl } \\
\text { transferase }\end{array}$ & bre-3 & HY483 & ye26 & Wild type & Wild type \\
\hline $\begin{array}{c}\beta-1,3 \text {-galactosyl } \\
\text { transferase }\end{array}$ & bre-5 & HY498 & ye17 & Suppressor & Suppressor \\
\hline $\begin{array}{l}\beta-1,4-\mathrm{N} \text {-acetyl- } \\
\text { galactosamin } \\
\text { yl transferase }\end{array}$ & bre-4 & HY485 & ye27 & Suppressor & Suppressor \\
\hline $\begin{array}{l}\beta \text {-glycosyl } \\
\text { transferase }\end{array}$ & bre-2 & HY494 & ye31 & Suppressor & Suppressor \\
\hline
\end{tabular}

Figure 2 Glucosylceramide (GlcCer) mediates clozapine-induced developmental delay/lethality and inhibition of pharyngeal pumping. The sphingolipid (SL) metabolic pathways conserved from yeast to humans is shown (Deng et al, 2008; Griffitts et al, 2005). Enzymes in red indicates hypersensitive to clozapine, blue indicates resistant to clozapine and black indicates the same as wild type. The results of clozapine-induced developmental delay/lethality assays and pharyngeal pumping assays on all the mutants of enzymes involved in SL metabolism are shown. For the pharyngeal pumping assays, $n=10, t$-test is performed, and regarded to be significant from wild type at $p<0.05$. 

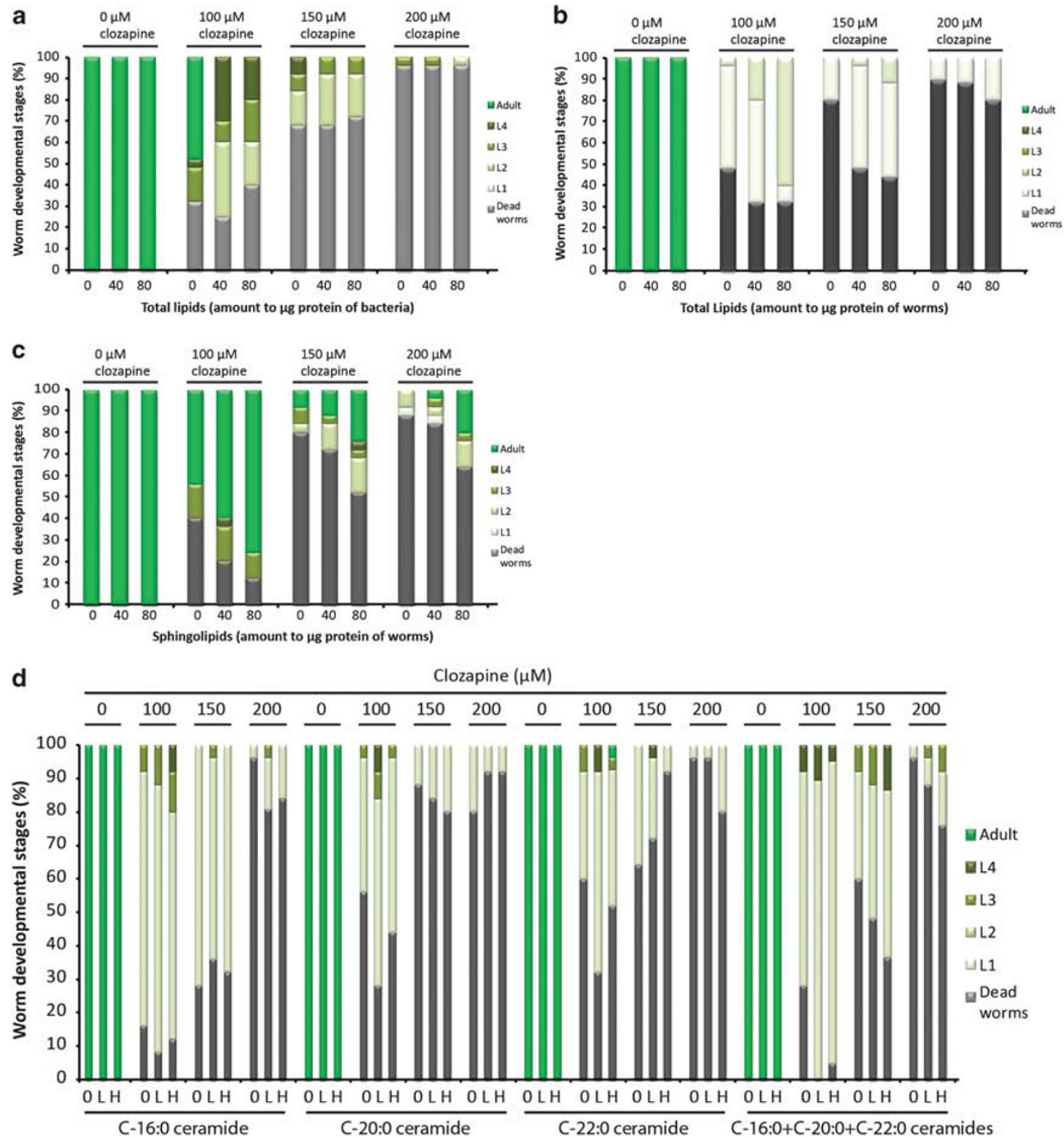

Figure 3 Rescue of hyl-I mutant using extracted total lipids, sphingolipids (SLs), and ceramides. (a-c) The hyl-I (ok976) mutant worms grown at 0, I00, I 50, and 200 MM clozapine are supplemented with total lipids extracted from the OP50 bacteria (a) and the N2 worms (b) or sphingolipids extracted from the N2 worms (c), all amounting to 0, 40, and $80 \mu g$ protein of starting materials. (d) The hyl- I (ok976) mutant worms grown at 0, 100, I50, and 200 $\mu M$ clozapine are supplemented with $0(0), 15(\mathrm{~L})$, and $30 \mu \mathrm{l}(\mathrm{H}) \mathrm{C}-\mathrm{I}$ 6:0, C-20:0, and C-22:0 ceramide or combination of them.

higher (Figures $5 \mathrm{f}-\mathrm{h}$ ), indicating that GlcCer might be able to mediate the formation of autophagosomes.

\section{DISCUSSION}

\section{Analysis of Components of SL Metabolism Identifies GlcCer as a Potential Mediator of Some of the Effects of Clozapine}

We show evidence demonstrating that GlcCer is altered by clozapine and may mediate some effects of clozapine. Using the clozapine-induced developmental delay/lethality model and the inhibition of pharyngeal pumping phenotypes, we analyzed over 10 mutants of genes centered on ceramide metabolism and discovered that enzymes that impact upon GlcCer levels mediate some of effects of clozapine. These findings were made possible by use of C. elegans, which has many available mutants (Deng et al, 2008). Analysis of a single gene mutant (eg, only sms-1), in a complex metabolic network, may not reveal the key molecules responsible for the disease. Comprehensive analysis of all the components in a complex metabolic network, as reported above, can provide an effective means to determine the molecules responsible for a metabolic disease or a drug effect.

\section{GlcCer Negatively Regulates Autophagy}

Ceramide triggers autophagy by interfering with the activation of Akt/PKB upstream of mTOR (Scarlatti et al, 2004). It 

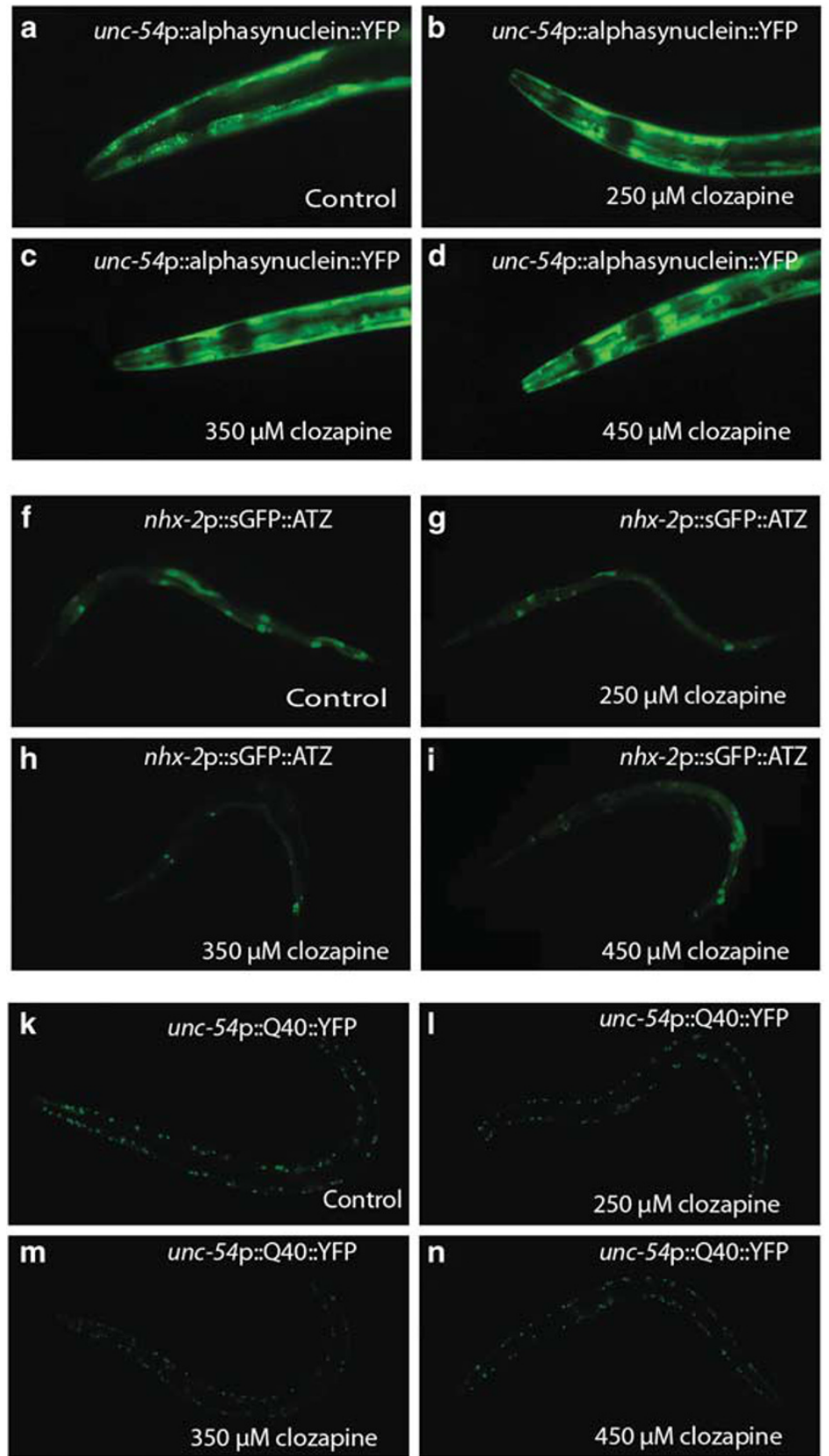
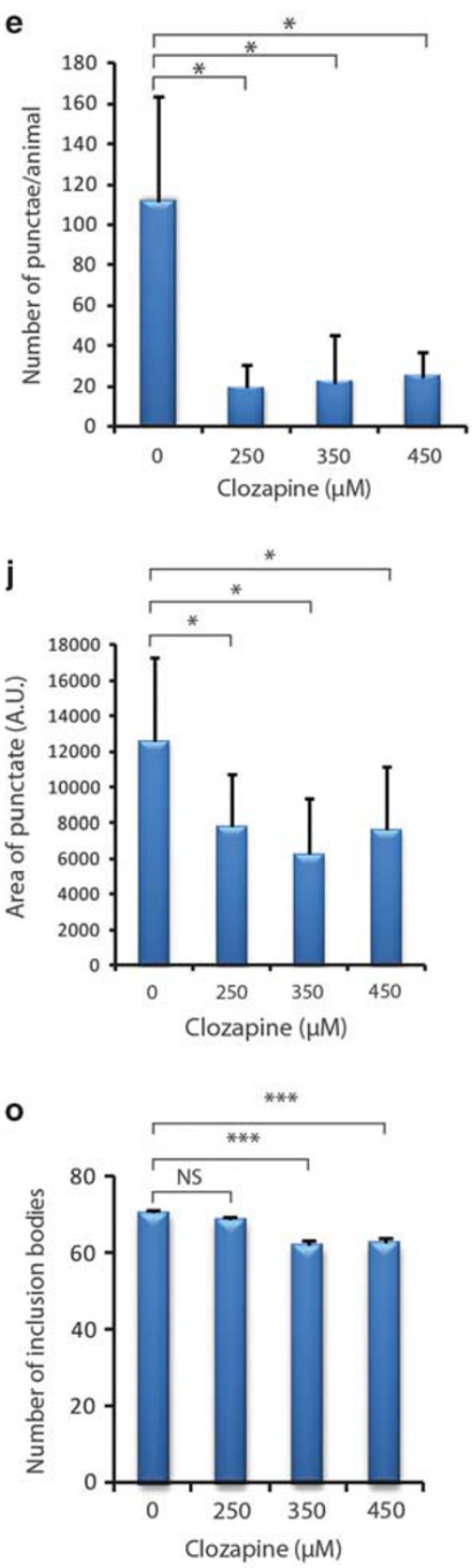

Figure 4 Clozapine reduces protein aggregations. (a-e) The wild-type worm carrying the unc-54p:: $\alpha$-synuclein::yfp transgene, where the $\alpha$-synuclein labeled with YFP in muscles driven by the unc-54 promoter, were treated with clozapine at $0 \mu \mathrm{M}$ (a), $250 \mu \mathrm{M}$ (b), $350 \mu \mathrm{M}$ (c), and $450 \mu \mathrm{M}$ (d) were observed under fluorescence microscopy. The puncta were quantified and shown in (e). (f-j) The wild-type worms carrying the nhx-2p::sGFP::ATZ transgene, where the ATZ labeled with the GFP marker in the pharynx and intestines driven by the $n h x-2$ promoter, were treated with clozapine at $0 \mu M(f), 250 \mu M(g), 350 \mu M(h)$, and 450 HM (i) were observed under fluorescence microscopy. The puncta were quantified and shown in (j). (k-o) The wild-type worms carrying the unc-54p: Q40::yfp transgene, where the PolyQ labeled with the YFP marker in muscles driven by the unc-54 promoter, were treated with clozapine at $0 \mu \mathrm{M}(\mathrm{k}), 250 \mathrm{\mu M}$ (I), $350 \mu \mathrm{M}(\mathrm{m})$, and $450 \mu \mathrm{M}(\mathrm{n})$ were observed under fluorescence microscopy. The inclusion bodies were quantified and shown in (o). In (e, j, and o), $n=10$, $t$-test is performed, and $* p<0.05$, **** $p<0.001$.

also activates JNK1 to phosphorylate Bcl-2, which alleviates the inhibitory effect of Bcl-2 on autophagy (Pattingre et al, 2009). Such an effect of ceramide could be responsible for ceramide-induced cell death (Daido et al, 2004; Li et al, 2009). However, this effect might be different from what we have found concerning GlcCer-regulated autophagy, where GlcCer may regulate autophagy in a negative manner (Figure 5). In line with this finding, the loss of two ceramide-synthesizing enzymes hyl-1 and lagr-1 extends the life span of C. elegans in an autophagosome-dependent manner (Mosbech et al, 2013). In addition, in cultured mammalian cells, inhibition of GlcCer synthase stimulated autophagy flux in neurons (Shen et al, 2014). The glucose moiety appears necessary for such an effect, as indicated by our finding that mutants of $s r f-3$, coding for a nucleotide sugar transporter used to transport glucose from the 

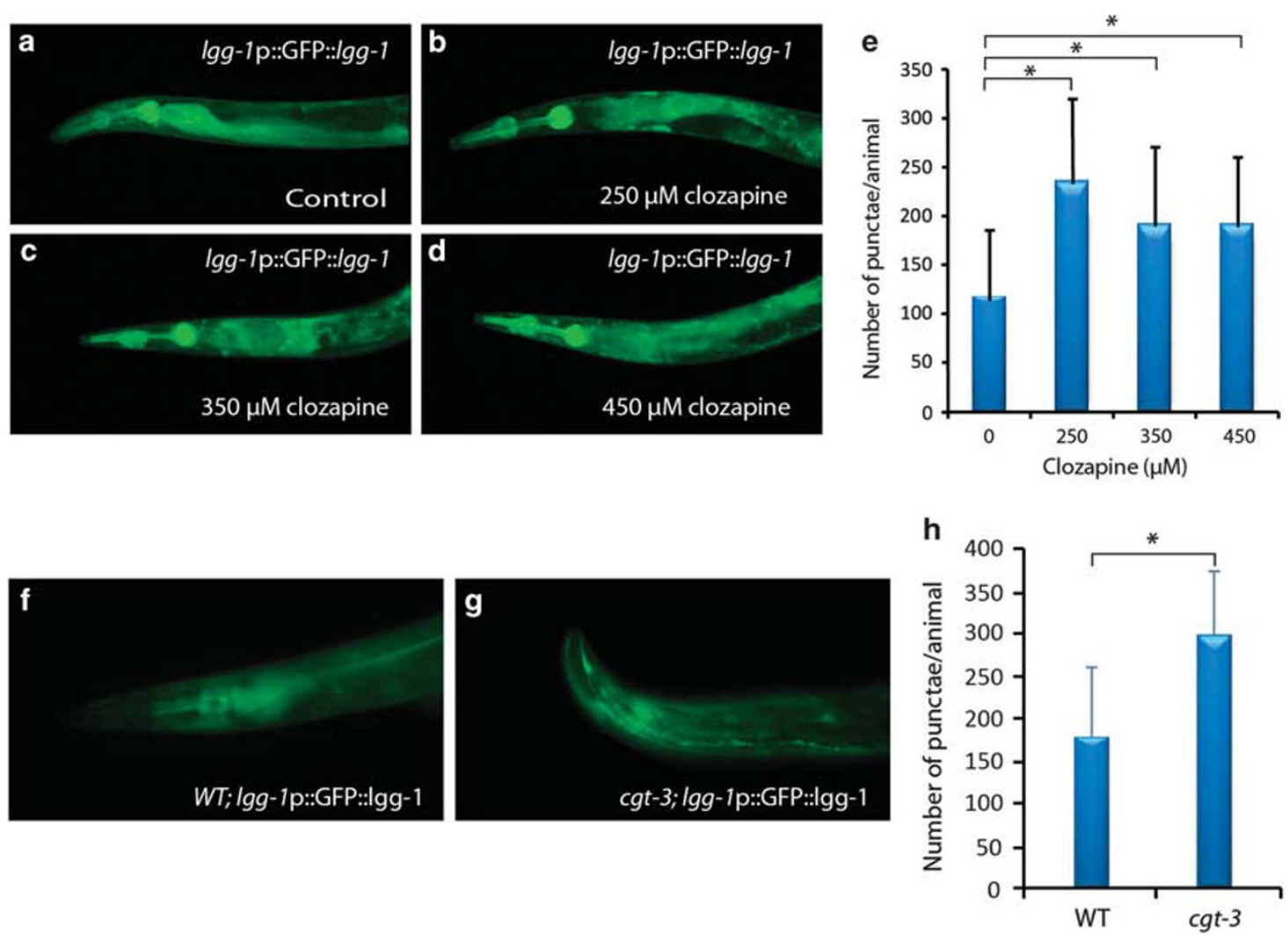

Figure 5 Clozapine enhances autophagosome formation, which could be regulated by glucosylceramide. (a-e) The wild-type worms carrying the lgg-I p:: gfp::Igg-I transgene, where the autophagosome component LGG-I, labeled with the GFP marker in the pharynx and intestines, driven by the unc-54 promoter, were treated with clozapine at $0 \mu \mathrm{M}$ (a), $250 \mu \mathrm{M}$ (b), $350 \mu \mathrm{M}$ (c), and $450 \mu \mathrm{M}$ (d) were observed under fluorescence microscopy. The inclusion bodies were quantified and shown in (e). (f-h) The expression of the lgg-I marker in the pharynx and intestines, driven by the unc-54 promoter, in wild type ( $\mathrm{f}$ ) and cgt-3(tm504) (g) were observed under fluorescence microscopy. The puncta were quantified and shown in (h). In (e and $h), n=10, t$-test is performed, and $* p<0.05$.

cytoplasm into endoplasm reticulum, are hypersensitive to clozapine (Figure 1). It could be interesting to explore if the decrease of glucose, a substrate of GlcCer, results in lower levels of GlcCer.

GlcCer accumulates in Gaucher disease patients because of mutations in GBA1. Gaucher disease patients have an elevated risk of Parkinson's disease and PD is associated with mutations of $\alpha$-synuclein. Shen et al (2014) demonstrated that inhibition of glucosylceramide synthase reduces levels of $\alpha$-synuclein and stimulates autophagy flux by inhibiting AKT-mTOR signaling, and increasing the number and size of lysosomal/late endosomal structures, which correlates with our finding that lower levels of glucosylceramide enhances the number of autophagosomes (Figure 5). However, inhibition of autophagy did not modify the decrease in $\alpha$-synuclein, suggesting that there may be an inhibitor-insensitive autophagy pathway (Shen et al, 2014). Glucosylceramide was also found to be involved in determining the apical polarity of C. elegans intestine and the sorting of new components to the expanding apical membrane (Zhang et al, 2011). Therefore, the mechanisms by which glucosylceramide negatively regulates autophagosomes and the clearing or processing of protein aggregates still need to be clarified.

\section{Some APDs Increase Clearance of Aggregated Proteins}

In a screen for drugs targeting tau neurotoxicity, using $C$. elegans as a model, McCormick et al (2013) found that the typical antipsychotic azaperone was able to reduce levels of insoluble tau and thus provide protection against neurodegeneration (McCormick et al, 2013). Additionally, Li et al (2014) identified fluphenazine as able to reduce proteotoxicity in a screen of the Library of Pharmacologically Active Compounds using C. elegans strains carrying the $\alpha 1$ antitrypsin deficiency transgene. They further found that fluphenazine enhanced the rate of intracellular degradation of ATZ and reduced the cellular ATZ load in mammalian cell line models; and in the PiZ mouse model, fluphenazine reduced the accumulation of ATZ in the liver and mediated a decrease in hepatic fibrosis (Li et al, 2014). These observations, in combination with our finding that clozapine reduces multiple protein aggregations (Figure 4), demonstrate that APDs may increase the clearance of protein aggregations, although there is a caveat that the marker proteins may be also reduced. APD-induced clearance of protein aggregations may be produced by lowering levels of glucosylceramide. However, as also noted, some of the effect may be mediated through lysosomes. That is, APD may have effects on both digestive compartments. 


\section{Ceramides in Schizophrenia and other Brain Diseases}

In a study using high-throughput mass spectrometry to profile white and gray matter lipid levels in the prefrontal cortex, ceramide levels were significantly increased in the white matter of schizophrenia and bipolar patients, although ceramide levels were not influenced by antipsychotic treatment (Schwarz et al, 2008). The total ceramide fraction of the stratum corneum lipids in first-episode antipsychoticnaïve schizophrenia patients was significantly reduced with inverse alterations of several single ceramide classes compared with matched controls (Smesny et al, 2013). In addition to these biochemical findings, the expression levels of the genes involved in SL metabolism were found to be altered. Narayan et al (2009) have observed that seven related genes (UGT8, SGPP1, GALC, B4GALT6, SPTC2, ASAH1, and GAL3ST1) in early-stage schizophrenia patients displayed a decrease in expression levels (Narayan et al, 2009). These studies suggest that there is an association between ceramide and schizophrenia, although more work needs to be carried out to reveal their precise relationships. Additionally, ceramide may be relevant to other brain diseases (BenDavid and Futerman, 2010). Plasma ceramide and glucosylceramide metabolism is altered in sporadic Parkinson's disease (Mielke et al, 2013), and particular species of serum ceramides are associated with incident Alzheimer disease (Mielke et al, 2012).

\section{Implication in Mammals}

Our findings linking clozapine and autophagy may have implications in mammals, including humans. Autophagy has an important role in the central nervous system by contributing to neuronal homeostasis. Autophagic failure has been linked to neurologic dysfunction and a variety of neurodegenerative disease, as well as the pathogenesis of schizophrenia. A recent work showed that there is a significant reduction in mRNA levels of a crucial autophagy-related protein, beclin 1 (BECN1), in the hippocampus of patients with schizophrenia (MerenlenderWagner et al, 2015). The activity-dependent neuroprotective protein (ADNP), a binding partner of LC3 that is a key component of the autophagic machinery, exhibits increased levels in both the brain and circulating lymphocytes in schizophrenia (Merenlender-Wagner et al, 2015). Administration of NAP, a peptide fragment of ADNP, enhances the activity of ADNP-LC3, a key component of the autophagic machinery interaction and reverses the decrease in hippocampal BECN1 mRNA levels in a mouse model of schizophrenia. The combination of NAP with clozapine results in complete normalization of behavioral outcomes (Merenlender-Wagner et al, 2014).

To summarize, we have observed that clozapine regulates GlcCer and, consistent with past studies, that GlcCer negatively regulates autophagy. Owing to the important role of ceramides in apoptosis and the emerging role of glucosylceramide in some genetic diseases and in neurodegeneration, these findings may have relevance to the use of APDs or more selectively targeted derivatives of APDs in seeking new treatment methods of these diseases. Last, there is a literature on phospholipid abnormalities in psychotic disorders, and the findings may be important in understanding some of the mechanisms by which APDs have antipsychotic effects. We realize that the work presented only begins to address these issues, and that the mechanisms underlying the effects of clozapine on ceramides and of clozapine and ceramides on autophagy require more study, but we believe the results may help suggest new areas of research on existing drugs and suggest novel approaches for the development of new drugs.

\section{FUNDING AND DISCLOSURE}

The work in our laboratory was supported by an NIH Clinical Scientist Development award, K08NS002083, funds of the Program for Neuropsychiatric Research, at McLean Hospital, and a NARSAD Young Investigator Award to Edgar A Buttner. AH Futerman is the Joseph Meyerhoff Professor of Biochemistry at the Weizmann Institute of Science. Bruce M Cohen is the Robertson-Steele Professor of Psychiatry at Harvard Medical School. The authors declare no conflict of interest.

\section{ACKNOWLEDGMENTS}

L Hao, EA Buttner, BM Cohen, O Ben-David, and AH Futerman designed experiments and interpreted the results; L Hao and O Ben-David performed experiments; L Hao, SM Babb, BM Cohen, O Ben-David, and AH Futerman drafted the manuscript. We thank Yuji Kohara for sms-1 cDNA clones, Jeb Gaudet for plasmids and worm strains of gland cell markers, Jean-Claude Martinou for the hyl-1 plasmid, and Min Han for two worm strains. We also thank Mitani Shoehei for some mutant worm strains. We thank Craig Hunter and his lab members who allowed us to use their microinjection apparatus before we set up our own, and Donna McPhie, Dost Öngör, Christopher W Cowan, Sabina Berreta, Vadim Bolshakov, and their lab members for their help and for sharing instruments and materials. Some strains were provided by the CGC, which is funded by the NIH Office of Research Infrastructure Programs (P40 OD010440). The preliminary quantification of SLs was performed by the Lipidomics Core at the Medical University of South Carolina, where research is supported, in part, by the Lipidomics Shared Resource, Hollings Cancer Center, Medical University of South Carolina (P30 CA138313), and the Lipidomics Core in the SC Lipidomics and Pathobiology COBRE (P20 RR017677). This work is dedicated to the memory of Dr Edgar (Ned) A Buttner, who passed away on 15 October 2015, for his inspiring enthusiasm, warm collegiality, and excellence in science.

\section{REFERENCES}

Barrows BD, Haslam SM, Bischof LJ, Morris HR, Dell A, Aroian RV (2007). Resistance to Bacillus thuringiensis toxin in Caenorhabditis elegans from loss of fucose. J Biol Chem 282: 3302-3311.

Ben-David O, Futerman AH (2010). The role of the ceramide acyl chain length in neurodegeneration: involvement of ceramide synthases. Neuromol Med 12: 341-350.

Brunello N, Masotto C, Steardo L, Markstein R, Racagni G (1995). New insights into the biology of schizophrenia through the mechanism of action of clozapine. Neuropsychopharmacology 13: 177-213. 
Crilly J (2007). The history of clozapine and its emergence in the US market: a review and analysis. Hist Psychiatry 18: 39-60.

Daido S, Kanzawa T, Yamamoto A, Takeuchi H, Kondo Y, Kondo S (2004). Pivotal role of the cell death factor BNIP3 in ceramideinduced autophagic cell death in malignant glioma cells. Cancer Res 64: 4286-4293.

Deng X, Yin X, Allan R, Lu DD, Maurer CW, Haimovitz-Friedman A et al (2008). Ceramide biogenesis is required for radiation-induced apoptosis in the germ line of C. elegans. Science 322: 110-115.

Donohoe DR, Aamodt EJ, Osborn E, Dwyer DS (2006). Antipsychotic drugs disrupt normal development in Caenorhabditis elegans via additional mechanisms besides dopamine and serotonin receptors. Pharmacol Res 54: 361-372.

Donohoe DR, Jarvis RA, Weeks K, Aamodt EJ, Dwyer DS (2009). Behavioral adaptation in $C$. elegans produced by antipsychotic drugs requires serotonin and is associated with calcium signaling and calcineurin inhibition. Neurosci Res 64: 280-289.

Dwyer DS, Aamodt E, Cohen B, Buttner EA (2014). Drug elucidation: invertebrate genetics sheds new light on the molecular targets of CNS drugs. Front Pharmacol 5: 177.

Fribourg M, Moreno JL, Holloway T, Provasi D, Baki L, Mahajan R et al (2011). Decoding the signaling of a GPCR heteromeric complex reveals a unifying mechanism of action of antipsychotic drugs. Cell 147: 1011-1023.

Futerman AH (2015). Preface to the special issue on brain lipids. Biochim Biophys Acta 1851: 997-998.

Futerman AH, Stieger B, Hubbard AL, Pagano RE (1990). Sphingomyelin synthesis in rat liver occurs predominantly at the cis and medial cisternae of the Golgi apparatus. J Biol Chem 265: 8650-8657.

Gault CR, Obeid LM, Hannun YA (2010). An overview of sphingolipid metabolism: from synthesis to breakdown. $A d v$ Exp Med Biol 688: 1-23.

Gohlke JM, Dhurandhar EJ, Correll CU, Morrato EH, Newcomer JW, Remington G et al (2012). Recent advances in understanding and mitigating adipogenic and metabolic effects of antipsychotic drugs. Front Psychiatry 3: 62.

Gosai SJ, Kwak JH, Luke CJ, Long OS, King DE, Kovatch KJ et al (2010). Automated high-content live animal drug screening using $C$. elegans expressing the aggregation prone serpin alpha1antitrypsin Z. PLoS One 5: e15460.

Griffitts JS, Haslam SM, Yang T, Garczynski SF, Mulloy B, Morris H et al (2005). Glycolipids as receptors for Bacillus thuringiensis crystal toxin. Science 307: 922-925.

Gulbins E, Palmada M, Reichel M, Luth A, Bohmer C, Amato D et al (2013). Acid sphingomyelinase-ceramide system mediates effects of antidepressant drugs. Nat Med 19: 934-938.

Hao L, Buttner EA (2014). Methods for studying the mechanisms of action of antipsychotic drugs in Caenorhabditis elegans. J Vis Exp 84: e50864.

Harvald EB, Olsen AS, Faergeman NJ (2015). Autophagy in the light of sphingolipid metabolism. Apoptosis 20: 658-670.

Jernigan PL, Hoehn RS, Grassme H, Edwards MJ, Muller CP, Kornhuber J et al (2015). Sphingolipids in major depression. Neurosignals 23: 49-58.

Kang C, Avery L (2009). Systemic regulation of starvation response in Caenorhabditis elegans. Genes Dev 23: 12-17.

Karmacharya R, Lynn SK, Demarco S, Ortiz A, Wang X, Lundy MY et al (2011). Behavioral effects of clozapine: involvement of trace amine pathways in C. elegans and M. musculus. Brain Res 1393 : 91-99.

Karmacharya R, Sliwoski GR, Lundy MY, Suckow RF, Cohen BM, Buttner EA (2009). Clozapine interaction with phosphatidyl inositol 3-kinase (PI3K)/insulin-signaling pathway in Caenorhabditis elegans. Neuropsychopharmacology 34: 1968-1978.

Kim MK, Kim SH, Yu HS, Park HG, Kang UG, Ahn YM et al (2012). The effect of clozapine on the AMPK-ACC-CPT1 pathway in the rat frontal cortex. Int J Neuropsychopharmacol 15: 907-917.

Lewis JA, Fleming JT (1995). Basic culture methods. Methods Cell Biol 48: 3-29.

Li DD, Wang LL, Deng R, Tang J, Shen Y, Guo JF et al (2009). The pivotal role of c-Jun $\mathrm{NH} 2$-terminal kinase-mediated Beclin 1 expression during anticancer agents-induced autophagy in cancer cells. Oncogene 28: 886-898.

Li J, Pak SC, O'Reilly LP, Benson JA, Wang Y, Hidvegi T et al (2014). Fluphenazine reduces proteotoxicity in C. elegans and mammalian models of alpha-1-antitrypsin deficiency. PLoS One 9: e87260.

Lieberman JA, Bymaster FP, Meltzer HY, Deutch AY, Duncan GE, Marx CE et al (2008). Antipsychotic drugs: comparison in animal models of efficacy, neurotransmitter regulation, and neuroprotection. Pharmacol Rev 60: 358-403.

Lukasiewicz S, Faron-Gorecka A, Kedracka-Krok S, DziedzickaWasylewska M (2011). Effect of clozapine on the dimerization of serotonin $5-\mathrm{HT}(2 \mathrm{~A})$ receptor and its genetic variant $5-\mathrm{HT}(2 \mathrm{~A})$ H425Y with dopamine $\mathrm{D}(2)$ receptor. Eur J Pharmacol 659: 114-123.

Marza E, Simonsen KT, Faergeman NJ, Lesa GM (2009). Expression of ceramide glucosyltransferases, which are essential for glycosphingolipid synthesis, is only required in a small subset of C. elegans cells. J Cell Sci 122(Part 6): 822-833.

McCormick AV, Wheeler JM, Guthrie CR, Liachko NF, Kraemer BC (2013). Dopamine D2 receptor antagonism suppresses tau aggregation and neurotoxicity. Biol Psychiatry 73: 464-471.

Menuz V, Howell KS, Gentina S, Epstein S, Riezman I, FornallazMulhauser $\mathrm{M}$ et al (2009). Protection of C. elegans from anoxia by HYL-2 ceramide synthase. Science 324: 381-384.

Merenlender-Wagner A, Malishkevich A, Shemer Z, Udawela M, Gibbons A, Scarr E et al (2015). Autophagy has a key role in the pathophysiology of schizophrenia. Mol Psychiatry 20: 126-132.

Merenlender-Wagner A, Shemer Z, Touloumi O, Lagoudaki R, Giladi E, Andrieux A et al (2014). New horizons in schizophrenia treatment: autophagy protection is coupled with behavioral improvements in a mouse model of schizophrenia. Autophagy 10: 2324-2332.

Mielke MM, Bandaru VV, Haughey NJ, Xia J, Fried LP, Yasar S et al (2012). Serum ceramides increase the risk of Alzheimer disease: the Women's Health and Aging Study II. Neurology 79: 633-641.

Mielke MM, Maetzler W, Haughey NJ, Bandaru VV, Savica R, Deuschle C et al (2013). Plasma ceramide and glucosylceramide metabolism is altered in sporadic Parkinson's disease and associated with cognitive impairment: a pilot study. PLoS One 8: e73094.

Mosbech MB, Kruse R, Harvald EB, Olsen AS, Gallego SF, Hannibal-Bach HK et al (2013). Functional loss of two ceramide synthases elicits autophagy-dependent lifespan extension in $C$. elegans. PLoS One 8: e70087.

Narayan S, Head SR, Gilmartin TJ, Dean B, Thomas EA (2009). Evidence for disruption of sphingolipid metabolism in schizophrenia. J Neurosci Res 87: 278-288.

Narayan S, Thomas EA (2011). Sphingolipid abnormalities in psychiatric disorders: a missing link in pathology? Front Biosci (Landmark edn) 16: 1797-1810.

Nomura KH, Murata D, Hayashi Y, Dejima K, Mizuguchi S, Kage-Nakadai E et al (2011). Ceramide glucosyltransferase of the nematode Caenorhabditis elegans is involved in oocyte formation and in early embryonic cell division. Glycobiology 21: 834-848.

Pattingre S, Bauvy C, Levade T, Levine B, Codogno P (2009). Ceramide-induced autophagy: to junk or to protect cells? Autophagy 5: 558-560.

Saur T, DeMarco SE, Ortiz A, Sliwoski GR, Hao L, Wang X et al (2013). A genome-wide RNAi screen in Caenorhabditis elegans 
identifies the nicotinic acetylcholine receptor subunit ACR-7 as an antipsychotic drug target. PLoS Genet 9: e1003313.

Scarlatti F, Bauvy C, Ventruti A, Sala G, Cluzeaud F, Vandewalle A et al (2004). Ceramide-mediated macroautophagy involves inhibition of protein kinase B and up-regulation of beclin 1 . J Biol Chem 279: 18384-18391.

Schmitt A, Wilczek K, Blennow K, Maras A, Jatzko A, Petroianu G et al (2004). Altered thalamic membrane phospholipids in schizophrenia: a postmortem study. Biol Psychiatry 56: 41-45.

Schwarz E, Prabakaran S, Whitfield P, Major H, Leweke FM, Koethe D et al (2008). High throughput lipidomic profiling of schizophrenia and bipolar disorder brain tissue reveals alterations of free fatty acids, phosphatidylcholines, and ceramides. J Proteome Res 7: 4266-4277.

Shen W, Henry AG, Paumier KL, Li L, Mou K, Dunlop J et al (2014). Inhibition of glucosylceramide synthase stimulates autophagy flux in neurons. J Neurochem 129: 884-894.

Sidransky E (2005). Gaucher disease and parkinsonism. Mol Genet Metab 84: 302-304.
Smesny S, Schmelzer CE, Hinder A, Kohler A, Schneider C, Rudzok M et al (2013). Skin ceramide alterations in first-episode schizophrenia indicate abnormal sphingolipid metabolism. Schizophr Bull 39: 933-941.

Sohlenkamp C, Geiger O (2016). Bacterial membrane lipids: diversity in structures and pathways. FEMS Microbiol Rev 40: 133-159.

Virmani A, Pinto L, Bauermann O, Zerelli S, Diedenhofen A, Binienda ZK et al (2015). The carnitine palmitoyl transferase (CPT) system and possible relevance for neuropsychiatric and neurological conditions. Mol Neurobiol 52: 826-836.

Wang X, Piccolo CW, Cohen BM, Buttner EA (2014). Transient receptor potential melastatin (TRPM) channels mediate clozapine-induced phenotypes in Caenorhabditis elegans. J Neurogenet 28: 86-97.

Zhang H, Abraham N, Khan LA, Hall DH, Fleming JT, Gobel V (2011). Apicobasal domain identities of expanding tubular membranes depend on glycosphingolipid biosynthesis. Nat Cell Biol 13: 1189-1201.

Supplementary Information accompanies the paper on the Neuropsychopharmacology website (http://www.nature.com/npp) 